\title{
Circulating makorin ring finger protein 3 levels decline in boys before the clinical onset of puberty
}

\author{
Tero Varimo1, Leo Dunkel2 ${ }^{2}$ Kirsi Vaaralahti ${ }^{3}$, Päivi J Miettinen ${ }^{1,4}$, Matti Hero ${ }^{1, *}$ and \\ Taneli Raivio ${ }^{1,3}$,*
}

${ }^{1}$ Children's Hospital, University of Helsinki and Helsinki University Hospital, Finland, 2William Harvey Research Institute, Barts and the London School of Medicine and Dentistry, Queen Mary University of London, London, UK, ${ }^{3}$ Faculty of Medicine/ Physiology, University of Helsinki, Helsinki, Finland, and ${ }^{4}$ Research Programs Unit, Molecular Neurology, and Biomedicum Stem Cell Center, University of Helsinki, Finland

*(M Hero and T Raivio contributed equally to this work)

Correspondence should be addressed to T Raivio

Email

taneli.raivio@helsinki.fi

\section{Abstract}

Objective: Makorin ring finger protein 3 (MKRN3) gene restrains the hypothalamic-pituitary-gonadal axis.

In girls, peripheral levels of MKRN3 decline prior to the onset of puberty. We described longitudinal changes in serum MKRN3 levels in boys before and during puberty and assessed the effect of inhibition of estrogen biosynthesis on MKRN3 levels.

Design: Longitudinal serum samples from a double-blind, randomized controlled study in 30 boys (age range: 9.1-14.2 years) with idiopathic short stature who received placebo $(\mathrm{Pl} ; n=14)$ or aromatase inhibitor letrozole (Lz; $2.5 \mathrm{mg} /$ day; $n=16$ ) for 2 years.

Methods: We analyzed the relationships between serum MKRN3 and clinical and biochemical markers of puberty by using summary measures.

Results: Serum MKRN3 declined by $669 \pm 713 \mathrm{pg} / \mathrm{mL}$ per year $(P<0.001)$. This change was biphasic, as the levels decreased during Tanner genital stage G1 $(-2931 \pm 2750 \mathrm{pg} / \mathrm{mL}$ per year) and plateaued thereafter $(-560 \pm 1510 \mathrm{pg} / \mathrm{mL}$ per year) $(P<0.05)$. During G1, MKRN3 levels in Lz-treated subjects decreased slower than in Pl-treated boys $(-782 \pm 3190$ vs $-2030 \pm 821 \mathrm{pg} / \mathrm{mL}$ per year, $P<0.05)$. The decrease in serum MKRN3 levels in $\mathrm{G} 1$ was associated with increases in LH $(r=-0.5, P<0.01)$, testosterone $(r=-0.6, P<0.01)$, and inhibin $\mathrm{B}(r=-0.44, P<0.05)(n=26)$.

Conclusion: Peripheral MKRN3 levels in boys appear to serve as a readout of the diminishing central inhibition that controls the onset of puberty.

\section{Introduction}

The recent discovery of paternally inherited loss-offunction mutations in makorin ring finger protein 3 (MKRN3) as a cause of central precocious puberty (CPP) suggests a pivotal role for MKRN3 in the transrepression of sexual maturation, but the exact mechanism remains unclear $(1,2)$. In mice, hypothalamic Mkrn3 expression is downregulated toward the onset of puberty (1). In humans, MKRN3 is expressed not only in the hypothalamus (3) but also in other tissues (4). There are currently two publications on circulating MKRN3 levels in humans $(5,6,7)$. In girls, MKRN3 levels declined before the onset of puberty and correlated negatively with increasing gonadotropin levels (5), which further underpins the potential role of MKRN3 in the central inhibitory control of puberty. In adult men, circulating MKRN3 levels did not seem to reflect the integrity of the hypothalamic-pituitary-gonadal (HPG) axis $(6,7)$, as the levels were similar in men with hypogonadal or normal testosterone levels. 
In this work, we describe longitudinal changes in MKRN3 levels in boys before and after the onset of puberty. To test the hypothesis that peripheral MKRN3 levels are modulated by estrogen, we compared MKRN3 levels between boys treated with aromatase inhibitor letrozole (Lz, a blocker of estrogen biosynthesis) and placebo (Pl) (8).

\section{Subjects and methods}

Thirty boys with idiopathic short stature (ISS) were recruitedinadouble-blind, randomized, placebo-controlled trial that investigated aromatase inhibitor therapy in the treatment of ISS (8). Sixteen boys were treated with $\mathrm{Lz}$ $(2.5 \mathrm{mg} /$ day) for 2 years and 14 received Pl. The study protocol together with the characterization and the hormonal markers of the patients has been reported previously (8). In short, the subjects were investigated 6 -monthly for 2 years and at the final visit, 36 months after the beginning of the study. At baseline, the mean age was 11 years (range 9.1-14.2 years) and Tanner genital stage was prepubertal (G1) in 28, G2 (increase in testicular volume above $3 \mathrm{~mL}$ ) in one, and G3 in one boy $(8,9)$. Six boys (20\%) had delayed puberty, defined as G1 at the age of 14 years or older. During the 2 years of the treatment, 8 out of 16 and 8 out of 14 boys showed physical signs of puberty progression (G2 or more) in Lz and Pl groups respectively. The visit at 36 months indicated that 7 out of 10 and 6 out of 11 boys were pubertal. Testicular volumes were calculated by using the formula: length $\times$ width $^{2} \times 0.52$ (10). Chronic and endocrine diseases underlying short stature were excluded. A written informed consent was obtained from all participants and their guardians. The study protocol was approved by the ethics committee of the Helsinki University Hospital, Helsinki, Finland, and the Finnish Medicines Agency (FIMEA).

\section{Hormonal analyses}

Blood samples were taken before $1000 \mathrm{~h}$ every 6 months for 2 years and at the final visit at 36 months, and sera were stored at -20 or $-80^{\circ} \mathrm{C}$ until hormonal analyses were performed. Serum testosterone, gonadotropin, and inhibin B levels were determined as reported (8). Serum MKRN3 levels were quantified by using the commercially available Human MKRN3 ELISA kit (Lot L08OMK24) (MyBioSource, San Diego, CA, USA). In brief, prior to the assay, all samples were diluted (in 1:8) to bring the analyte level to the measuring range of the assay, as instructed by the manufacturer. The final results were achieved by multiplying the results by the dilution factor. In $5 \%$ $(8 / 160)$ of the diluted samples, however, the MKRN3 levels were still slightly $(n=6)$ or clearly $(n=2)$ higher than the highest standard of the assay $(2000 \mathrm{pg} / \mathrm{mL})$. These extrapolated values were accepted, because human serum diluted linearly from $\sim 6800 \mathrm{pg} / \mathrm{mL}$ to the measuring range of the assay. As reported by the manufacturer, the sensitivity of the assay is $7.8 \mathrm{pg} / \mathrm{mL}$. Longitudinal samples from each individual were assayed on the same assay plate as singleton measurements (within-assay coefficient of variation $(\mathrm{CV})$ was $6 \%$ and between-assay $\mathrm{CV}$ was $9.2 \%)$.

\section{Statistical analysis}

The data are presented as mean \pm standard deviation (s.D.) unless otherwise informed. SPSS statistical software for Windows, release 22.0 (SPSS) was used for statistical analyses. The longitudinal changes in the levels of MKRN3, gonadotropins, testosterone, and inhibin B were investigated by using summary measures (11). In brief, we fitted a linear regression line for each boy, with age as an independent variable and circulating MKRN3, testosterone, $\mathrm{LH}, \mathrm{FSH}$, or inhibin B levels as a dependent variable. Then the regression coefficients were analyzed with the $t$-test or used in correlation analysis. Withingroup comparisons were assessed with Mann-Whitney $U$ test and linear correlations with Spearman's rank correlation. All tests were two sided and $P$-values $<0.05$ were considered statistically significant.

\section{Results}

During the follow-up, serum MKRN3 declined by $669 \pm 713 \mathrm{pg} / \mathrm{mL}$ per year $(P<0.001)$. This overall decrease was similar between boys treated with $\mathrm{Lz}(n=16)$ $(-657 \pm 750 \mathrm{pg} / \mathrm{mL}$ per year) and those who received $\mathrm{Pl}$ $(n=14)(-683 \pm 690 \mathrm{pg} / \mathrm{mL}$ per year) $(P=\mathrm{NS})$. However, as $M K R N 3$ restrains the HPG axis, we evaluated the changes in circulating MKRN3 levels before and during the clinical onset of puberty, and therefore adjusted MKRN3 levels according to G2. The levels of MKRN3 appeared to decline before G2, with less change in G2 and thereafter (Fig. 1A). Indeed, in 13 boys with available data before and after the clinical onset of puberty (G2), MKRN3 levels declined faster during $\mathrm{G} 1$ than after entering puberty $(-2931 \pm 2750$ vs $560 \pm 1510 \mathrm{pg} / \mathrm{mL}$ per year, $n=13$, $P<0.05$ respectively) (Fig. 1B). Then we investigated whether the change in MKRN3 was modulated by Lz. Surprisingly, MKRN3 levels during G1 declined faster in Pl-treated boys $(n=14)$ than in Lz-treated subjects $(n=14)$ 
A
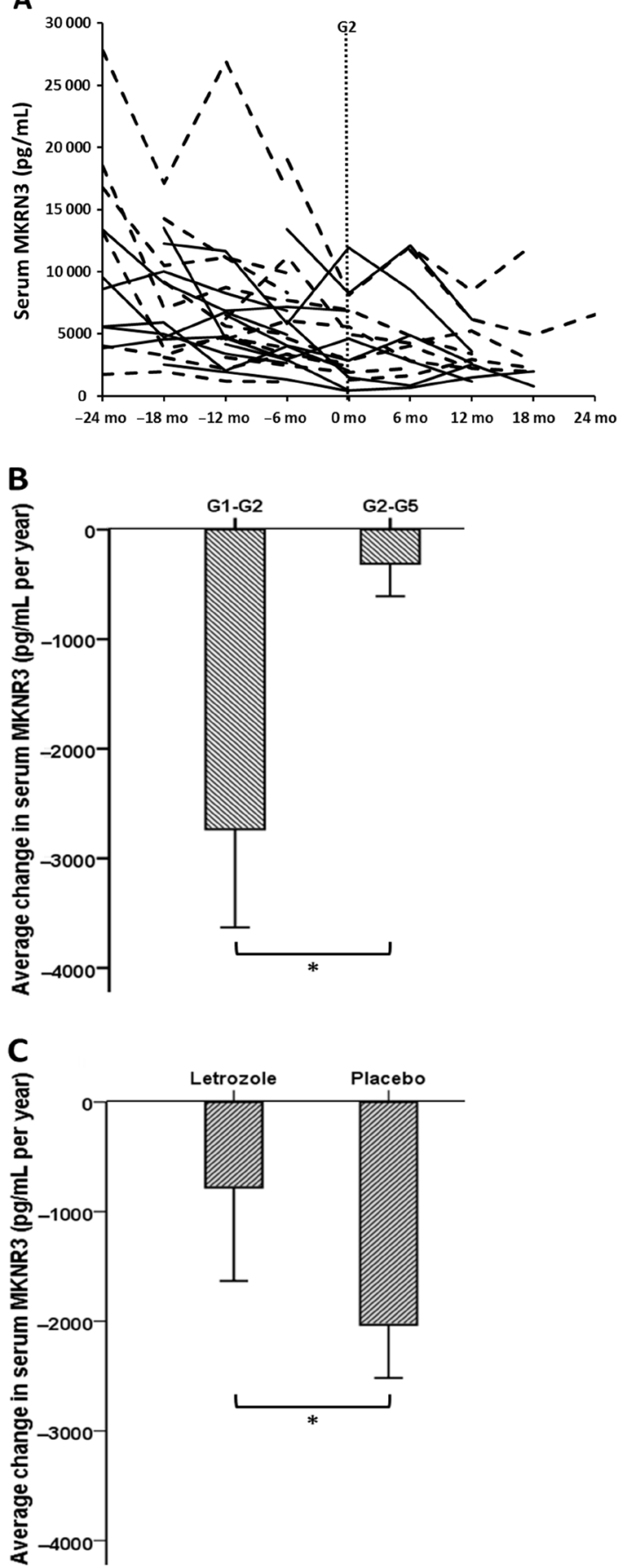

Figure 1

(A) Longitudinal changes in serum MKRN3 levels before Tanner stage G2 (mean age 12.9, range 10.4-15.3years) and thereafter in 30 boys with ISS. Sixteen boys received Lz (dashed line) and 14 received $\mathrm{PI}$ (black line). (B) The rate of decline in serum MKRN3 levels before (G1) and after entering puberty (G2-G5) in 13 boys with ISS. (C) The rate of decline in serum MKRN3 levels in Lz-treated boys $(n=14)$ and in those who received $\mathrm{PI}(n=14)$ during Tanner genital stage $1 .{ }^{*} P<0.05$.

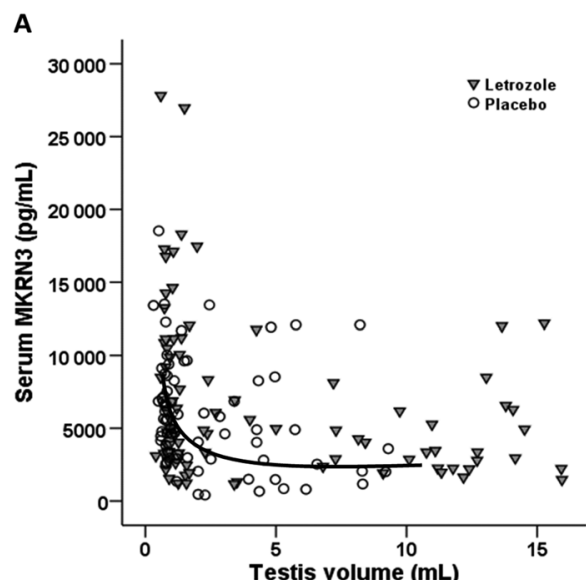

B
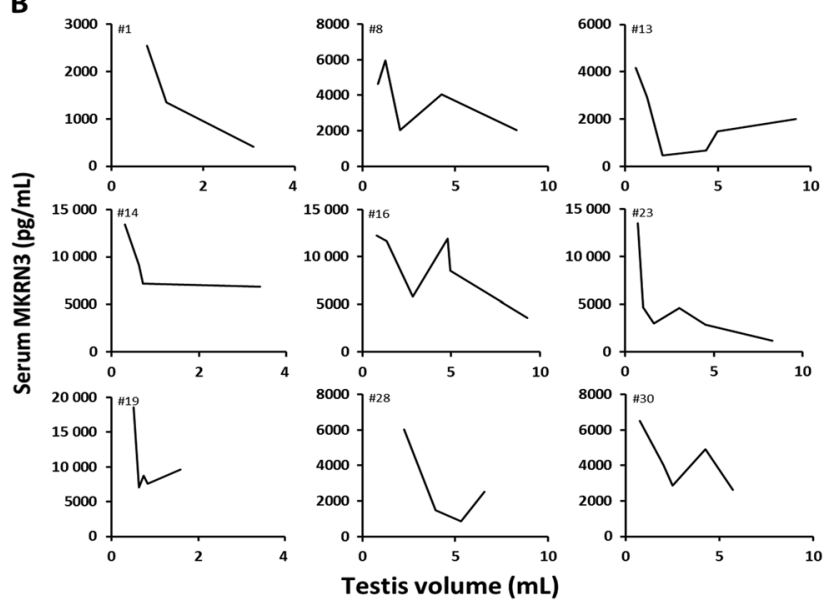

Figure 2

(A) The relationship between serum MKRN3 levels and testicular volume (TV) $(r=-0.3, P<0.001)$ in 160 samples from 30 boys with ISS during a 3-year follow-up. The line represents the association between TV and MKRN3 in boys treated with placebo $(r=-0.4, P<0.001)$. (B) Individual changes in serum MKRN3 levels and testis volumes in nine boys with ISS who received placebo.

$(-2030 \pm 1821$ vs $-782 \pm 3190$, respectively, $P<0.05)$ (Fig. 1C). During the course of puberty, serum MKRN3 levels correlated with testis volumes $(r=-0.3, P<0.001)$ with a rapid MKRN3 decline in early puberty (Fig. 2A). This reciprocal relationship is illustrated in Fig. $2 \mathrm{~B}$ for nine individuals. To further investigate the relationship between the clinical onset of puberty (i.e. increase in testis volume) and circulating MKRN3, we identified the highest and the lowest individual MKRN3 values for each boy who progressed from G1 to G2 $(n=22)$. In these boys, the highest MKRN3 values $(9192 \pm 5541 \mathrm{pg} / \mathrm{mL})$ were observed when their average testicular volume was $2.0 \pm 2.9 \mathrm{~mL}$, and the lowest MKRN3 levels $(4480 \pm 3943 \mathrm{pg} / \mathrm{mL})$ 
were measured later in puberty, when the average testicular volume was significantly higher $5.8 \pm 4.5 \mathrm{~mL}(P<0.001)$.

Then we investigated the relationship between serum MKRN3 and gonadotropin, testosterone and inhibin B in G1. Indeed, during G1, the rate of change in MKRN3 correlated with the rate of change in testosterone $(r=-0.4, n=28, P<0.05)$, but not with the corresponding changes in other hormone levels $(P=\mathrm{NS})$. After an exclusion of two boys who exhibited an increase in MKRN3 level, the rate of MKRN3 change during G1 correlated with the change in LH $(r=-0.5, P<0.01$, $n=26)$, inhibin B $(r=0.44, P<0.05, n=26)$ and strongly with testosterone $(r=0.6, P<0.01, n=26)$. The reciprocal changes between serum MKRN3 and LH levels before the clinical onset of puberty in boys $6,8,13,14,17$, and 23 are shown in Fig. 3. Placebo- and Lz-treated boys had similar testis volumes and levels of testosterone, inhibin $\mathrm{B}$, and gonadotropins in G1. In addition, no clinical or biochemical evidence for differences in pubertal timing was observed (8).

Delayed maturation is common in boys with ISS, and six boys fulfilled the conventional criteria of delayed puberty. One of these boys had the highest levels of MKRN3, whereas four boys exhibited serum MKRN3 within the range of those who entered puberty before 14 years of age. A significant difference in the rate of MKRN3 decline was found between boys with delayed onset and normal timing of puberty $(-81 \pm 612 \mathrm{vs}-812 \pm 671 \mathrm{pg} / \mathrm{mL}$ per year, respectively, $P<0.05)$. However, we found no significant correlations between indices of pubertal timing (age at G2 and the degree of bone age delay calculated as calendar age minus bone age at the beginning) and MKRN3 levels (data not shown).

\section{Discussion}

Our longitudinal data show that MKRN3 levels in boys decline before the clinical onset of puberty and that this decrease is associated with the activation of the HPG axis. These findings are in agreement with the results of the recent study in girls (5). In boys, however, the change in MKRN3 levels appears biphasic with a rapid decline prior to clinical onset of puberty and slower changes thereafter once puberty progresses, whereas in girls, the decline in serum MKRN3 continued to mid-puberty (5). The reason for this difference is not known. Another sex-dependent difference in MKRN3-related mechanisms that regulates the onset of puberty is suggested by the finding that boys with CPP due to a paternally inherited mutation in MKRN3 appear to have a smaller decline in the age of puberty onset than girls (for review, see (12)). These findings suggest that there are sex-specific differences in
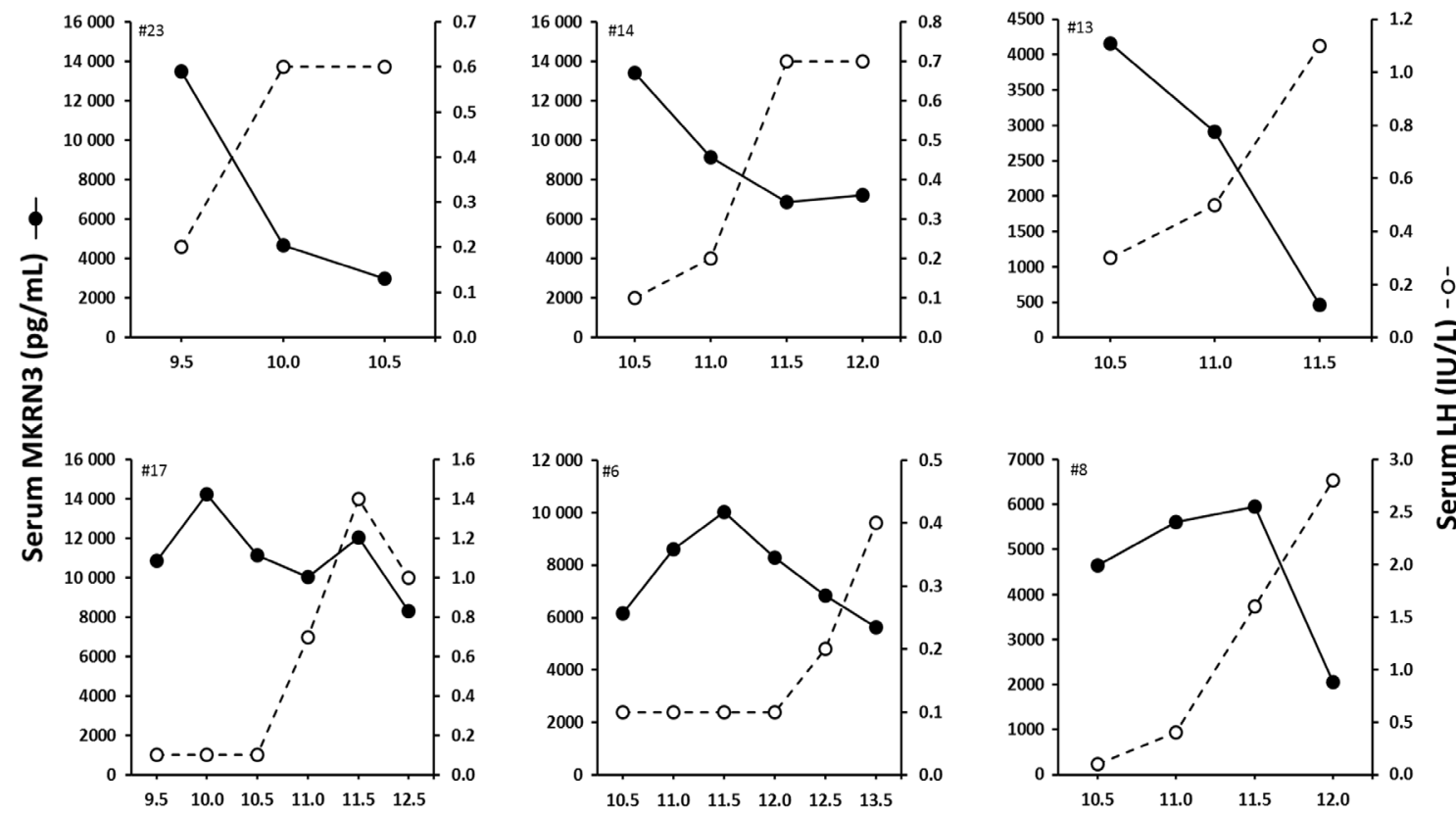

Age (years)

Figure 3

Serum MKRN3 and LH levels in six boys with ISS during peripuberty. 
the relative role of MKRN3 in the central inhibitory control of puberty onset, which is still inadequately understood. Studies carried out in humans and rhesus monkeys show that the central inhibition of gonadotropin secretion is more pronounced in males than in females (for review, see (13)). Consistently, idiopathic CPP is more common in girls than in boys $(1,12,14,15)$.

The ISS boys in the current work received aromatase inhibitor Lz in an attempt to increase adult height (8). In Lz-treated boys, the rate of prepubertal decline in MKRN3 was slower compared with those who received placebo. On the one hand, this finding lends credence to the hypothesis that MKRN3 is involved in the wellknown advancing effect of sex steroids on the timing of puberty (16). It also offers a potential explanation for the synchrony between skeletal and HPG axis maturation, as a strong association exists between bone age - a measure of estrogen exposure - and pubertal timing (16). On the other hand, providing that circulating MKRN3 levels reflect the function of the puberty clock, one would have expected later puberty in Lz-treated boys than in those who received Pl, but such a difference was not found (8). However, a subtle difference between the groups may have escaped detection. It is also possible that the modulation of MKRN3 levels by Lz was compensated by other factors involved in the central control of sexual maturation. Finally, there are no reports on the relationship between estradiol and peripheral MKRN3 levels in humans. In girls, however, serum MKRN3 declines before and during puberty (5), which raises the possibility that estradiol negatively regulates MKRN3 secretion. This interaction would also explain the slower decline in serum MKRN3 in Lz-treated boys. Clearly, MKRN3 is not the only factor that affects pubertal timing as, for example, paternally inherited mutations in MKRN3 result in a relatively mild advancement of pubertal timing, especially in boys (2). Although MKRN3 levels in healthy men and in men with congenital hypogonadotropic hypogonadism $(\mathrm{CHH})$ did not differ $(6,7)$, serum MKRN3 may have a clinical value in the often notoriously difficult differential diagnosis between constitutional delay of growth and puberty and $\mathrm{CHH}$ (17), given that the delayed onset of puberty was associated with slow decline in MKRN3. It should be noted that this study has a relatively small sample size, which may reduce statistical power. Although MKRN3 levels were in agreement with the levels observed in healthy men $(6,7)$, the levels in boys showed high interindividual variation, which may reduce the clinical value of serum MKRN3 measurements. Similar high interindividual variation has been described in girls (5). Our results in boys are clearly higher than the previously reported levels in girls (5). However, we have not detected any major sex-dependent differences in serum MKRN3 levels between men and women, or between boys and girls (unpublished data). Thus, validation of the MKRN3 kit in larger studies and the use of international and national reference standards are expected to help in better understanding the fluctuations of circulating MKRN3 blood levels throughout life and possibly also between laboratories. Especially, studies on circulating MKRN3 levels in healthy boys and in boys with different conditions affecting the timing of puberty are needed to further validate the role of MKRN3 as a marker of the central control of the onset of puberty.

In conclusion, this is the first study to describe circulating MKRN3 levels in boys. Our results show that MKRN3 levels decline at the verge of puberty and that the levels are associated with the awakening of the HPG axis. Estrogen-lowering effect of $\mathrm{Lz}$ was associated with the peripubertal decline in MKRN3, but clinical correlates of these subtle changes could not be demonstrated. However, peripheral MKRN3 levels hold promises for improvements in our understanding of the complex mechanisms that regulate the timing of human puberty.

\section{Declaration of interest}

The authors declare that there is no conflict of interest that could be preceived as prejudicing the impartiality of the research reported.

\section{Funding}

This work was supported by the Academy of Finland (grant number 268356), Foundation for Pediatric Research (grant number 7495), and research funds of Helsinki University Hospital, Helsinki, Finland (grant number 2013101)

\section{References}

1 Abreu AP, Dauber A, Macedo DB, Noel SD, Brito VN, Gill JC, Cukier P, Thompson IR, Navarro VM, Gagliardi PC et al. Central precocious puberty caused by mutations in the imprinted gene MKRN3. New England Journal of Medicine 2013368 2467-2475. (doi:10.1056/ NEJMoa1302160)

2 Abreu AP, Macedo DB, Brito VN, Kaiser UB \& Latronico AC. A new pathway in the control of the initiation of puberty: the MKRN3 gene. Journal of Molecular Endocrinology 201554 R131-R139. (doi:10.1530/ JME-14-0315)

3 Kansakoski J, Raivio T, Juul A \& Tommiska J. A missense mutation in MKRN3 in a Danish girl with central precocious puberty and her brother with early puberty. Pediatric Research 201578 709-711. (doi:10.1038/pr.2015.159)

4 Uhlen M, Fagerberg L, Hallstrom BM, Lindskog C, Oksvold P, Mardinoglu A, Sivertsson A, Kampf C, Sjostedt E, Asplund A et al. Proteomics. Tissue-based map of the human proteome. Science 2015 347 1260419. (doi:10.1126/science.1260419)

5 Hagen CP, Sorensen K, Mieritz MG, Johannsen TH, Almstrup K \& Juul A. Circulating MKRN3 levels decline prior to pubertal onset 
and through puberty: a longitudinal study of healthy girls. Journal of Clinical Endocrinology and Metabolism 2015100 1920-1926. (doi:10.1210/jc.2014-4462)

6 Varimo T, Hero M, Kansakoski J, Vaaralahti K, Matikainen N \& Raivio T. Circulating makorin ring-finger protein-3 (MKRN3) levels in healthy men and in men with hypogonadotropic hypogonadism. Clinical Endocrinology 201584 638. (doi:10.1111/cen.12851)

7 Varimo T, Hero M, Kansakoski J, Vaaralahti K, Matikainen N \& Raivio T. Erratum to Circulating makorin ring-finger protein-3 (MKRN3) levels in healthy men and in men with hypogonadotropic hypogonadism. Clinical Endocrinology 201684 638. (doi:10.1111/ cen.13027)

8 Hero M, Norjavaara E \& Dunkel L. Inhibition of estrogen biosynthesis with a potent aromatase inhibitor increases predicted adult height in boys with idiopathic short stature: a randomized controlled trial. Journal of Clinical Endocrinology and Metabolism 2005 90 6396-6402.

9 Marshall WA \& Tanner JM. Variations in the pattern of pubertal changes in boys. Archives of Disease in Childhood 197045 13-231. (doi:10.1136/adc.45.239.13)

10 Hansen PF \& With TK. Clinical measurements of the testes in boys and men. Acta Medica Scandinavica Supplementum 1952266 457-465.

11 Matthews JN, Altman DG, Campbell MJ \& Royston P. Analysis of serial measurements in medical research. BMJ 1990300 230-235. (doi:10.1136/bmj.300.6719.230)

12 Simon D, Ba I, Mekhail N, Ecosse E, Paulsen A, Zenaty D, Houang M, Jesuran-Perelroizan M, De Filippo G, Salerno M et al. Mutations in the maternally imprinted gene MKRN3 are common in familial central precocious puberty. European Journal of Endocrinology 2015174 1-8. (doi:10.1530/EJE-15-0488)

13 Plant TM, Terasawa E \& Witchel SF. Puberty in non-human primates and man. In Knobil and Neill's Physiology of Reproduction, edn 4, ch 32, pp 1487-1536. Eds Plant TM \& Zeleznik AJ. Amsterdam, The Netherlands: Elsevier, 2015.

14 de Vries L, Gat-Yablonski G, Dror N, Singer A \& Phillip M. A novel MKRN3 missense mutation causing familial precocious puberty. Human Reproduction 201429 2838-2843. (doi:10.1093/humrep/ deu256)

15 Macedo DB, Abreu AP, Reis AC, Montenegro LR, Dauber A, Beneduzzi D, Cukier P, Silveira LF, Teles MG, Carroll RS et al. Central precocious puberty that appears to be sporadic caused by paternally inherited mutations in the imprinted gene makorin ring finger 3. Journal of Clinical Endocrinology and Metabolism 201499 E1097-E1103. (doi:10.1210/jc.2013-3126)

16 Flor-Cisneros A, Leschek EW, Merke DP, Barnes KM, Coco M, Cutler GB Jr \& Baron J. In boys with abnormal developmental tempo, maturation of the skeleton and the hypothalamic-pituitary-gonadal axis remains synchronous. Journal of Clinical Endocrinology and Metabolism 200489 236-241. (doi:10.1210/jc.2002-021954)

17 Boehm U, Bouloux PM, Dattani MT, de Roux N, Dode C, Dunkel L, Dwyer AA, Giacobini P, Hardelin JP, Juul A et al. Expert consensus document: European consensus statement on congenital hypogonadotropic hypogonadism - pathogenesis, diagnosis and treatment. Nature Reviews Endocrinology 201511 547-564. (doi:10.1038/nrendo.2015.112)

Received 6 December 2015

Revised version received 20 March 2016

Accepted 29 March 2016 